\title{
Further observations on inclusion-bearing cells in urinary sediment in infectious diseases
}

\author{
J. F. BOYD AND NADA NEDELKOSKA ${ }^{1}$ \\ From the Brownlee Laboratory, the University Department of Infectious Diseases, \\ Ruchill Hospital, Glasgow, N.W., and the University Department of Pathology, \\ Western Infirmary, Glasgow, $W .2$
}

SYNOPSIS A study of the cytology of the urinary sediment in 43 patients with known viral diseases has revealed a variety of inclusion-bearing cells in 28 .

The morphology of the cells suggest that the changes recorded may be due to the viral infections, at least in some instances, bearing in mind the findings of workers quoted in our 1964 report that cellular changes very similar to those induced by virus infections can be initiated by non-viral stimuli. Multinucleate giant cells are occasionally found in chickenpox, measles, herpes simplex infection, and in mumps.

The work of Bolande (1959 and 1961) stimulated us to examine the cytology of urinary sediment, and some of our preliminary observations have been recorded (Boyd and Nedelkoska, 1963 and 1964). These showed that inclusion-bearing cells were noted in 25 of 100 patients consecutively admitted to Ruchill Hospital, the 'positive' cases including five with varicella, two with herpes zoster, the other cases including central nervous system, respiratory, alimentary, and cutaneous conditions for which no viral aetiology was proved. Among the 75 'negative' cases, there were patients with illnesses of accepted viral aetiology, for example, rubella, primary herpes simplex, mumps, infective hepatitis, homologous serum jaundice, poliomyelitis (type 3 strain), and enteritis associated with ECHO virus type 2 infection. Although consideration ought to be given to the study of urinary sediment from normal (i.e. non-infected) children and adults, this investigation has not been pursued in view of the difficulty of defining normality. Appreciation of the existence of latent virus infections and of prolonged excretion of virus in the urine after some infections might make this line of pursuit less appropriate.

Our next approach therefore has been to examine a consecutive series of admissions with illnesses of accepted viral aetiology, and the results are presented in this article.

${ }^{1}$ British Council scholar. Present Address: The Clinic for Infectious Diseases, Skopje, Yugoslavia.

Received for publication 6 April 1967.

\section{MATERIAL AND METHODS}

A fresh specimen of urine was obtained from the patient on the first morning after admission to hospital and was treated in the same fashion as described in our earlier reports (Boyd and Nedelkoska, 1963 and 1964). Since these specimens were obtained usually about 7 a.m. and were processed between 10 and 11 a.m., the routine was established that the specimens were stored in Universal containers $(30 \mathrm{ml} ., 1 \mathrm{fl}$. oz.) at ward temperature rather than in a refrigerator. The heavy precipitation of urates and phosphates which tended to occur if the latter method was adopted seriously obscured the study of cells. Specimens which showed heavy bacterial growth during this period were discarded as being unsatisfactory; those showing only a few bacteria were retained. None of the patients included in this report belongs to the earlier series.

The criteria for 'positive' and 'negative' cells and for 'positive' and 'negative' urine samples are unchanged.

\section{RESULTS}

The results of the investigation are shown in the Table. Of 43 patients with illnesses of known viral aetiology, $28(65 \%)$ showed inclusion-bearing cells in the urine in varying numbers. This result differs very greatly from that of the earlier series. Our wider experience has shown that there is great variation in the cellularity of urine specimens even in a series of patients with the same viral illness, and we now appreciate that some patients exhibit abacterial pyuria during the course of virus illnesses, an 
TABLE

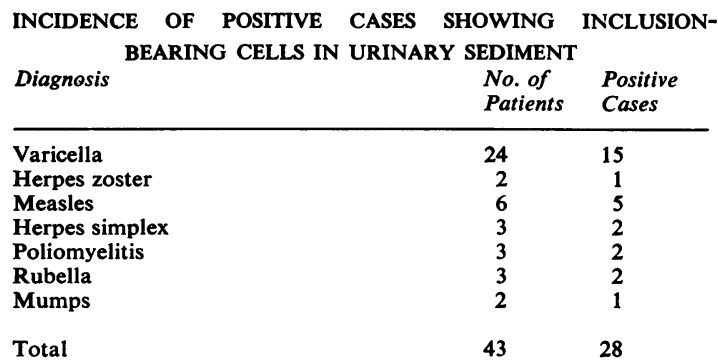

observation which has also been made by Hart and Cherry (1965).

There are 13 males and 11 females with varicella, their ages ranging from 3 months to 37 years, and 15 are positive. Only one of the nine negative cases showed no exfoliated cells in the urinary sediment. This group fails to support the consistently positive results of our earlier series.

Two more patients with herpes zoster, 64-year-old and 73-year-old females, are reported and only one urine specimen was positive. Cytologically it was a weak positive result. This finding is a marked contrast to the two examples quoted in our earlier reports. The discrepancy may be due partly to the condition remaining localized in the present examples. One of the earlier examples was also localized, however, but the urine sample was nevertheless moderately strongly positive.

The most persistently positive results are obtained in measles (morbilli), where five patients were positive of six examined (five males, one female), with the sixth case yielding an acellular specimen on admission.

There are three patients who are considered to have primary herpes simplex infections. Two are boys, aged $6 \frac{1}{4}$ yr. and $1 \frac{3}{4}$ yr., and a girl of 3 years. The girl and the older boy yielded virus from the lesions and showed good antibody responses. The younger boy, whose urine was acellular on admission and was therefore classified as negative, failed to yield herpes simplex virus from his lesions and serology was not performed.

The three patients with poliomyelitis, two girls of $3 \frac{1}{2}$ yr. and $5 \mathrm{yr}$., and a boy of $1 \frac{1}{4}$ yr., gave cellular urinary specimens on admission and the least cellular one, from the boy, was negative for inclusion-bearing cells. The other cases were weakly positive. The infection was due to polio-virus type 1 in all instances.

Of the three rubella patients, the negative specimen was from a 2-year-old girl whose admission urine sample was acellular. One of the two positive patients, a $2 \frac{1}{2}$-year-old boy, had been discharged from hospital three weeks earlier after recovery from chickenpox, and it is uncertain therefore $\stackrel{?}{?}$ whether or not his urinary inclusion-bearing cells were all due to rubella: it is possible that the recent $\frac{}{\bar{F}}$ chickenpox infection contributed partly to this $\frac{\bar{s}}{\vec{\sigma}}$ result.

One of the patients with mumps, not seen during the acute stage, was admitted with epididymoorchitis, and his urine was negative. Serology for mumps infection was not performed. The other $\vec{\omega}$ patient, an 18-year-old girl, was a typical case of $\stackrel{\odot}{\circ}$ mumps, both clinically and serologically. Her용 admission urine specimen was positive even although $\tilde{N}$ it was not very cellular.

The present series has given us further experience with the study of inclusion-bearing cells in urinary or sediment and the descriptions given in our 은 earlier series hold good for the present one. The most commonly affected cell is mononuclear, but $Z$

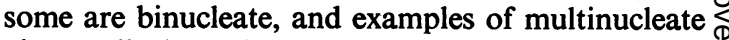
giant cells have been encountered in chickenpox, $\stackrel{Ð}{\rightrightarrows}$ measles, herpes simplex, and in mumps as described $\underset{\Phi}{ }$ below. The colours which are detailed relate to the original Papanicolaou-stained preparations and not $\mathscr{\odot}$ to the black and white illustrations printed here.

Figures 1 and 2 show cells encountered in one case of varicella. The first cell has the size and some of the features of a urinary tract transitional cell. In the rather ballooned nucleus there is a small spherical deeply blue-black body. Near the periphery $\mathbb{D}$ of the nucleus there is a smaller grey sphere, tenta- $\overrightarrow{\vec{B}}$ tively labelled a 'polar body', and between these two 3 structures there is a delta-shaped green intranuclear $\supset$ inclusion. The nucleoplasm is pale green. The position of the nuclear membrane is noticeable and is green. The cytoplasm is pale green and possesses? numerous tiny red inclusions of apparently equal size. The second cell possesses four ballooned nuclei although only three are in focus. Two show red $\frac{0}{3}$ intranuclear structures of rather irregular shape and

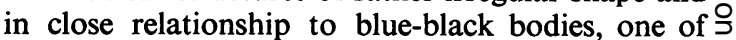
which is larger than the others and is ovoid. The $\rightarrow$ staining reactions are similar to the cell of Figure 1 . and this cell also shows numerous tiny red cyto- $N$ plasmic bodies of apparently equal size. This multinucleate giant cell is quite unlike the variety normally encountered in smears of vesicle fluid in chickenpox. $\omega$

Our studies of measles show that mononuclear and binucleate inclusion-bearing cells are encoun- 0 tered in the urine, thus confirming the observations $\mathbb{\varnothing}$ of Bolande, and that in addition, multinucleate ?+ giant cells, similar to the syncytial forms met with $\frac{0}{T}$ in the giant-cell pneumonia of measles, may be $\stackrel{+}{\mathbb{D}}$ noted occasionally, thus confirming observations $\odot$ made by Bolande (1959) and by Mitus, Enders, $\stackrel{\mathbb{Q}}{\Omega}$ Edsall, and Holloway (1965). Figures 3 and 4 show 


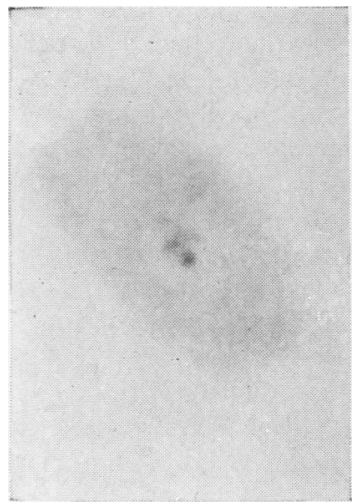

FIG. 1

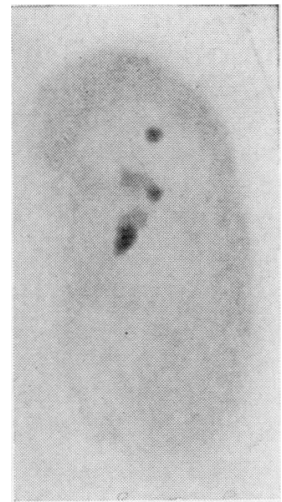

FIG. 2

FIGS. 1 and 2. Chickenpox. Papanicolaou. $\times 1,600$.

FIG. 3
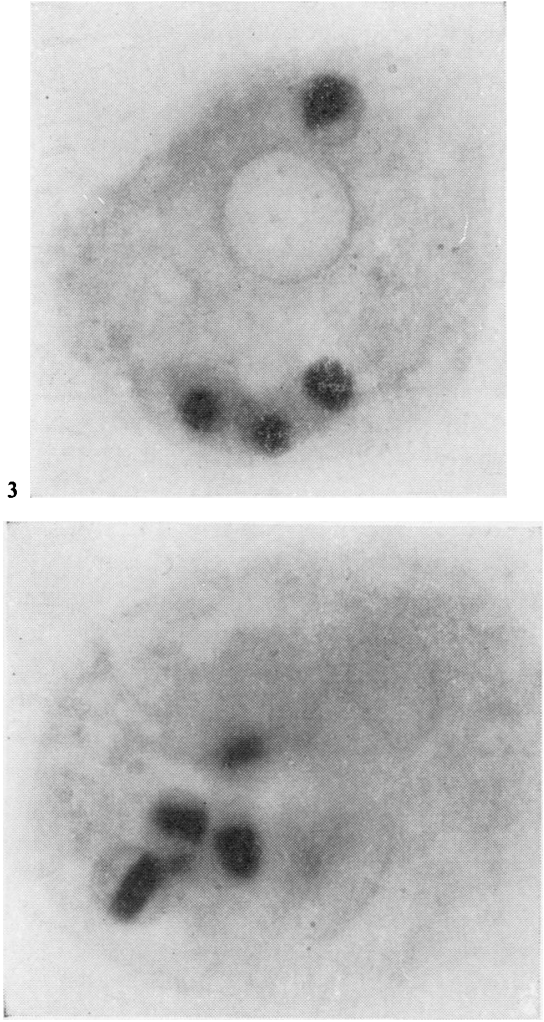

FIGS. 3 and 4. Measles. Papanicolaou. $\times 1,600$.

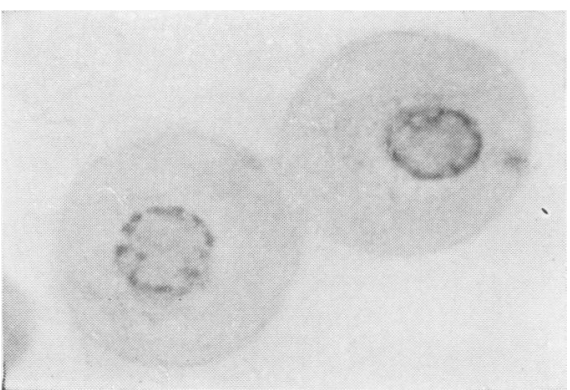

FIG. 5. H. simplex. Papanicolaou. $\times 1,300$.

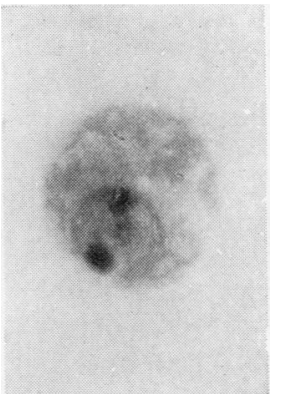

FIv. 0

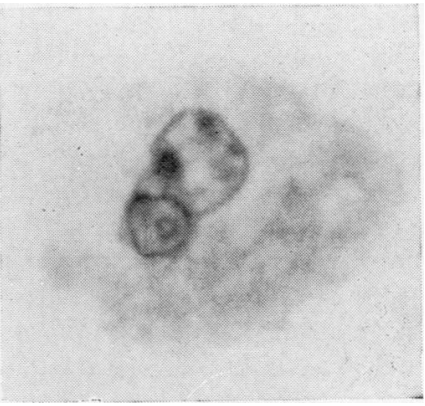

FIG. 7

FIGS. 6 and 7. H. simplex. Papanicolaou. $\times 1,300$.

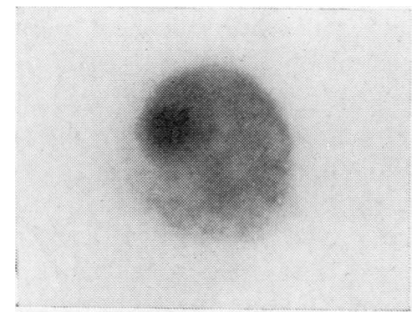

FIG. 8. Poliomyelitis. Papanicolaou. $\times 1,300$.

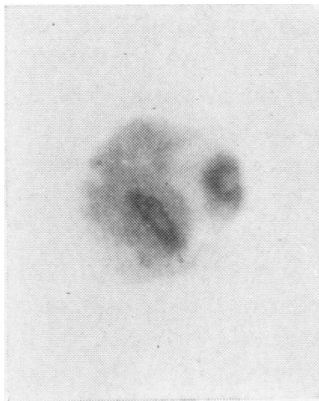

FIG. 9

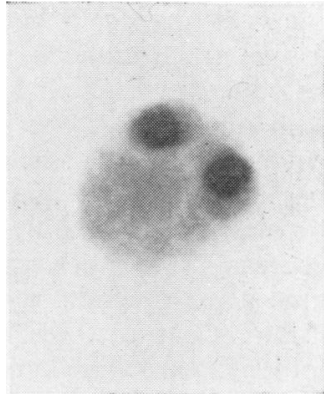

FIG. 10

FIGS. 9 and 10 . Rubella. Papanicolaou. $\times 1,300$.

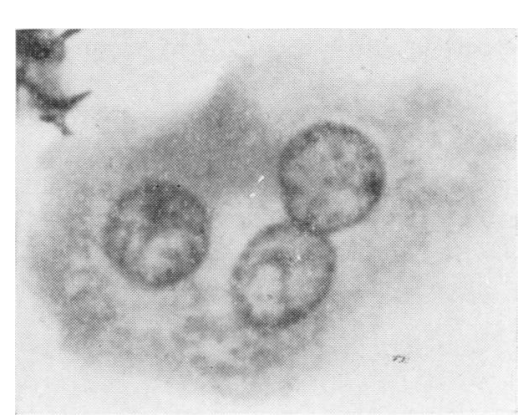

FIG. 11

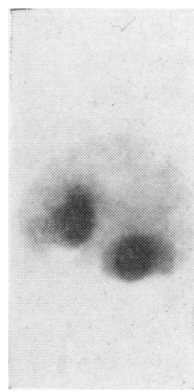

FIG. 12

FIGS. 11 and 12. Mumps. Papanicolaou. $\times 1,300$ 
two examples from the same patient. Figure 3 shows a large cell with four nuclei, all being virtually in focus. In each instance the nuclear chromatin forms a condensed blue-black solid sphere, and a dull red moderately enlarged spherical structure, considered to be the nucleolus, is present alongside. Within the surrounding homogeneously orange nucleoplasm there are a few small red and blue-black satellite structures not all of which are distinguishable in the illustration. It is uncertain if the position of the nuclear membrane is stained or whether the interface is the result of contrast staining reactions alone. The cytoplasm is moderately green and vacuolated peripherally, but it is red and granular centrally, and there is a biconcave spherical red inclusion which bears specks of unidentified refractile material at one or two points. The multinucleate giant cell of Fig. 4 possesses seven nuclei although only one is clearly in focus. Only two have been shown clearly to possess bodies which could be enlarged nucleoli; one is dull red, and the other is grey or very pale blue. The chromatin of each nucleus is condensed as a blue-black bar, sphere, or ovoid, and the nucleoplasm is clear and unstained in each instance. As in the case of the cell in Fig. 3, the limits of a nuclear membrane are well defined but whether or not the weak eosinophilia is genuine, or is a refractory artefact, is uncertain. The cytoplasm is red and granular and carries a red crescentic inclusion.

Figures 5, 6, and 7 are taken from a patient with herpes simplex infection and show different features. The cells in Fig. 5 bear a strong resemblance to urinary tract epithelial cells. They show somewhat ballooned nuclei, neither of which shows a nucleolus. Both nuclei show the blue-black chromatin to be condensed on the nuclear membrane as biconvex masses of almost equal size. In one instance, the distribution is moderately even, resembling a rosary, whereas in the other case it is disinctly uneven, and large gaps, resembling enlarged nuclear annuli, are visible. Centrally, the one nucleus shows a faint homogeneously stellate grey inclusion with clear incomplete halo, whereas the other nucleus shows a rather grey and white marbled nucleoplasm. The cytoplasm of one cell bears eosinophilic granules which are aggregated into masses of different size and shape, and the cytoplasm is pink centrally and green peripherally while the cytoplasm of the other cell is moderately green and bears occasional vacuoles.

Figure 6 shows a different situation. This mononuclear cell shows ballooning of the nucleus which is a feature of herpes simplex virus infection. Centrally, there is a deep red body, believed to be an enlarged nucleolus with a small red polar body alongside in clear nucleoplasm. The blue-black chromatin is condensed as large biconvex masses on a thin nuclear membrane on which there are also numerous very small, faintly staining biconvex masses. The cytoplasm is moderately green and vacuolated, and possesses a large orange cytoplasmic inclusion which is out of focus. There are several similar smaller inclusions on the cytoplasmic membrane. This particular cell shows some resemblance to cells from the urine sediment of a patient with $\vec{\circ}$ generalized zoster infection and illustrated in Figs. 3, 4, and 6 of Boyd and Nedelkoska (1964), but none of these cells show a red nucleolus or a red 'polar body'. The cell illustrated in Fig. 7 possesses three nuclei, but only two are in focus. All nuclei are ballooned. Two show no convincing nucleoli whereas the third (the smaller shown in Fig. 7) $\omega_{\tilde{N}}^{\infty}$ shows an unstained structure which might be nucleolus with a surrounding blue-black layer. Two nuclei show blue-black biconvex chromatin masses of varying sizes on a thickened nuclear membrane, whereas the third nucleus (which is out of focus) only shows a thickened nuclear membrane. The nucleoplasm of the nuclei shows areas of rarefaction. The cytoplasm is moderately green and vacuolated and shows many red inclusions some of which are arranged in a crescentic row.

Figure 8 shows a mononuclear cell from a patient with poliomyelitis and this cell shows a blue-black condensed sphere of chromatin in green homogeneous nucleoplasm. There are two red polar bodies, one or both of which may be nucleoli. Only one is in focus. While the position of the nuclear membrane is identifiable it is doubted if any staining is present. The cytoplasm is moderately green centrally, where there is a red paranuclear inclusion, and it is paler peripherally where there are several unstained vacuoles.

Mononuclear and binuclear inclusion-bearing cells have also been noted in rubella. Figure 9 shows $\delta$ one variety of binucleate cell. In each instance, the $₹$ nucleus appears to possess an unstained spherical 을 nucleolus, and the blue-black chromatin is condensed around this structure as a sphere in one case and as a cigar-shaped body in the other. The nucleoplasm is green, and the position of the nuclear membrane $O$ is shown although no nuclear membrane is visible. 0 Scanty small blue-black biconvex masses, however, N are present along the perimeter here and there. The 0 cytoplasm is green and an orange spherical inclusion is present although it is out of focus. Figure 10 is $\frac{2}{D}$ another binucleate cell showing essentially similar $\stackrel{?}{+}$ features. The nucleoli are unstained. The nucleo- 0 plasm is green. The cytoplasm is pale green and $\overrightarrow{0}$ granular and bears three moderate-sized red in- $\mathbb{D}$ clusions and many small ones.

Figure 11 shows a multinucleate giant cell in the 
urinary sediment of a patient with mumps. All three nuclei are ballooned and possess nucleoli which are vermilion red while two nucleoli also possess blue-black margins. The nuclear chromatin is blue-black or grey and stippled, and also forms small biconvex masses of varying size on thickened blue-black nuclear membranes. The nucleoplasm is clear and grey. The central nucleus shows the sex chromatin (or Barr body) against the nuclear membrane. The cytoplasm is grey, green and granular, with vacuolated areas, and showing small pinhead grey inclusions below the central nucleus. The binucleate cell in Fig. 12 shows unstained nucleoli with condensed blue-black nuclear chromatin. The surrounding nucleoplasm is deep green. No distinct nuclear membrane separates it from the grey-orange granular cytoplasm which is vacuolated and bears a moderate-sized homogeneous orange inclusion and several smaller ones.

\section{DISCUSSION}

In general, the present study shows that there are many varieties of fundamental change which can affect cells in the urinary sediment. We cannot state categorically that the changes recorded in any particular cell are the consequence of infection by the virus responsible for the patient's disease, although we consider that the results as a whole could be given such an interpretation.

Bolande (1959) noted inclusion-bearing cells in four urine specimens of five studied from patients with varicella but his description of the cell type is a general one and makes no reference to cells of the type shown in Figures 1 and 2. In their study of the cytopathology of parainfluenza type 3 virus infection in $\mathrm{HeLa}$ and monkey kidney cells in vitro, however, Love and Suskind (1961) show intranuclear inclusions of similar appearance to those shown in Figures 1 and 2.

In prodromal measles, multinucleate giant cell formations are encountered regularly in the lymphoid tissue throughout the body as the WarthinFinkeldey giant cell, and occasionally giant cells, are found in the lungs when pneumonia occurs. The giant cells in the former situation bear hundreds of pyknotic nuclei usually placed centrally in the cell which seldom shows cytoplasmic inclusions, while the syncytial forms in the lung alveoli classically show both intranuclear and cytoplasmic eosinophilic inclusions. The cells which are illustrated in Figures 3 and 4 resemble closely the syncytial giant cells even although eosinophilic intranuclear inclusions are absent. Sherman and Ruckle (1958) found a bladder mucosal giant cell in a patient with measles, although their illustration is not very convincing. Giant cells of the variety illustrated have not been noted in all patients with measles, however.

The descriptions of cells infected with herpes simplex virus mention that there is inhibition of mitosis, that the nuclei are ballooned, have lost their nucleoli, show margination of the nuclear chromatin on the nuclear membrane, and that there may be a large central inclusion which is usually eosinophilic and possesses a halo. It is clear, however, that this description, which applies both to the mononuclear as well as to the multinucleate giant cell, is only one instant in the course of a virus cycle in an infected cell. Love and Wildy (1963) have studied the ribonucleoproteins of $\mathrm{HeLa}$ cells infected with herpes simplex virus and have detailed many changes during the course of infection. One cell in Fig. 5 may show a classical A inclusion while the other cell may show A granules appearing within the $\mathrm{A}$ inclusion and, alongside, the two solitary blue-black spheres may be ' $\mathrm{B}$ ' bodies, as described by these authors. The large red 'nucleolus' in Fig. 6 may represent the pars amorpha of the nucleolus as described by early histologists, a term which has been employed effectively by Love in several publications in order to distinguish it from the nucleolini of the nucleolus, which appear to be invisible in the cell illustrated. The cell in Fig. 7 shows areas of rarefaction in the nucleoplasm, also described by Love and Wildy. It is debatable whether or not the smaller nucleus could be classified as a micronucleus, another feature recorded by Love and Wildy (1963) and more recently by Boiron, Tanzer, Thomas, and Hampe (1966) in tissue culture experiments.

As mentioned earlier, it was our impression that the urine samples from patients with poliomyelitis were not as strongly positive as, for example, were those from patients with measles. The cell illustrated in Fig. 8, although fixed and stained differently from the method employed on tissue cultures by Reissig, Howes, and Melnick (1956), appears to be similar to type 6 of their classification of progressive cytological changes after infection with poliovirus. Cowdry type B inclusions (1934) were not noted in this series, and this may be explained by the finding of Reissig et al. that type B inclusions are present about four hours after infection and are found in type 2 cells of their classification, that is to say, about halfway in the course of the virus cycle through the cell. Hart and Cherry (1965) noted inclusion-tearing cells in the urine of children after oral poliovirus vaccine and were rather uncertain about the significance of this finding. Abnormal urinary cytology was not found by Schultz and Neva (1966) in their experimental studies of poliovirus infections of mice and rats. 
One characteristic of earlier work on rubella grown on tissue cultures was the absence of any noticeable cytopathic effect, although more recent work (e.g., Veronelli and Maassab, 1965) appears to show that cytopathic effects can be produced if a suitable cell line is chosen. The above authors describe eosinophilic cytoplasmic inclusions in their experiments. Bolande (1959) reported finding inclusion-bearing cells in the urine of three patients with rubella out of four cases studied. Further, Heggie and Robbins (1964) reported the presence of inclusion-bearing cells in seven of 15 urine specimens from naval recruits with rubella while only one of 14 specimens from control subjects showed inclusion-bearing cells. We would not place any significance on the apparent difference between the incidence of positive results achieved by these authors and by ourselves. Our positive samples were not very strongly positive by our arbitrary criteria.

Utz and Szwed (1962) cultured mumps virus from 58 out of 110 urine specimens from 21 patients. Bolande (1959) reported inclusion-bearing cells in the urine of two patients with mumps and that the specimens were moderately positive. A solitary patient with mumps and submandibular abscess failed to reveal these cells in our previous publication
(Boyd and Nedelkoska, 1964) and only one of the two patients in this series was positive. We have been unable to find any evidence that other workers have noted multinucleate giant cells similar to that shown in Fig. 11 in the urine sediment of such patients.

We wish to thank W. Marshall F.I.M.L.T. for his technical assistance.

\section{REFERENCES}

Boiron, M., Tanzer, J., Thomas, M., and Hampe A. (1966). Nature (Lond.), 209, 737.

Bolande, R. P. (1959). Pediatrics, $24,7$.

(1961). New Engl.J. Med., 265, 919.

Boyd, J. F., and Nedelkoska, N. (1963). Scot. med. J., 8, 269. (1964). J. Path. Bact., 88, 115.

Cowdry, E. V. (1934). Arch. Path., 18, 527.

Hart, A. F., and Cherry, J. D. (1965). New Engl. J. Med., 272, 174. Heggie, A. D., and Robbins, F. C. (1964). Ibid., 271, 231.

Love, R., and Suskind, R. G. (1961). Exp. Cell Res., 24, 521. - and Wildy, P. (1963). J. Cell Biol., 17, 237.

Mitus, A., Enders, J. F., Edsall, G., and Holloway, A. (1965). Arch. ges. Virusforsch., 16, 331.

Reissig, M., Howes, D. W., and Melnick, J. L. (1956). J. exp. Med., $104,289$.

Schultz, I., and Neva, F. A. (1966). J. Immunol., 96, 74.

Sherman, F. E., and Ruckle, G. (1958). Arch. Path., 65, 587.

Utz, J. P., and Szwed, C. F. (1962). Proc. Soc. exp. Biol. (N.Y.) 110,841 .

Veronelli, J. A., and Maassab, H. F. (1965). Arch. ges. Virusforsch., $16,426$. 